\title{
Ref-1/APE1 Inhibition with Novel Small Molecules Blocks Ocular Neovascularization ${ }^{\mathrm{S}}$
}

\author{
(D) Sheik Pran Babu Sardar Pasha, ${ }^{1}$ 이 Kamakshi Sishtla, ${ }^{1}$ Rania S. Sulaiman, (1) Bomina Park,

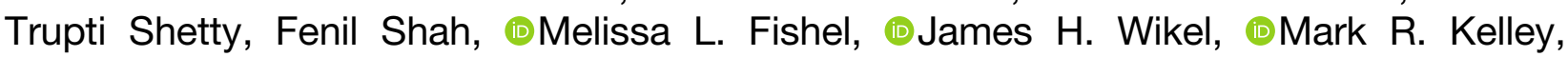 \\ and (1)Timothy W. Corson
}

\begin{abstract}
Eugene and Marilyn Glick Eye Institute, Department of Ophthalmology (S.P.B.S.P., K.S., R.S.S., B.P., T.S., T.W.C.), Department of Pharmacology and Toxicology (R.S.S., B.P., T.S., M.L.F., M.R.K., T.W.C.), Department of Biochemistry and Molecular Biology (M.R.K., T.W.C.), Herman B Wells Center for Pediatric Research, Department of Pediatrics (F.S., M.L.F., M.R.K.), and Melvin and Bren Simon Cancer Center (M.L.F., M.R.K., T.W.C.), Indiana University School of Medicine, Indianapolis, Indiana; and Apexian Pharmaceuticals (J.H.W.), Indianapolis, Indiana
\end{abstract}

Received February 7, 2018; accepted August 1, 2018

\section{ABSTRACT}

Ocular neovascular diseases like wet age-related macular degeneration are a major cause of blindness. Novel therapies are greatly needed for these diseases. One appealing antiangiogenic target is reduction-oxidation factor 1-apurinic/apyrimidinic endonuclease 1 (Ref-1/APE1). This protein can act as a redoxsensitive transcriptional activator for nuclear factor (NF) $-\kappa B$ and other proangiogenic transcription factors. An existing inhibitor of Ref-1's function, APX3330, previously showed antiangiogenic effects. Here, we developed improved APX3330 derivatives and assessed their antiangiogenic activity. We synthesized APX2009 and APX2014 and demonstrated enhanced inhibition of Ref-1 function in a DNA-binding assay compared with APX3330. Both compounds were antiproliferative against human retinal microvascular endothelial cells (HRECs; $\mathrm{Gl}_{50}$ APX2009: $1.1 \mu \mathrm{M}$, APX2014: $110 \mathrm{nM}$ ) and macaque choroidal endothelial cells (Rf/6a; $\mathrm{GI}_{50}$ APX2009: $26 \mu \mathrm{M}$, APX2014: $\left.5.0 \mu \mathrm{M}\right)$. Both compounds significantly reduced the ability of HRECs and $\mathrm{Rf} / 6 \mathrm{a}$ cells to form tubes at mid-nanomolar concentrations compared with control, and both significantly inhibited HREC and $\mathrm{Rf} / 6$ a cell migration in a scratch wound assay, reducing $\mathrm{NF}-\kappa \mathrm{B}$ activation and downstream targets. Ex vivo, APX2009 and APX2014 inhibited choroidal sprouting at low micromolar and high nanomolar concentrations, respectively. In the laserinduced choroidal neovascularization mouse model, intraperitoneal APX2009 treatment significantly decreased lesion volume by 4-fold compared with vehicle $(P<0.0001$, ANOVA with Dunnett's post-hoc tests), without obvious intraocular or systemic toxicity. Thus, Ref-1 inhibition with APX2009 and APX2014 blocks ocular angiogenesis in vitro and ex vivo, and APX2009 is an effective systemic therapy for choroidal neovascularization in vivo, establishing Ref-1 inhibition as a promising therapeutic approach for ocular neovascularization.

\section{Introduction}

Ocular neovascularization is the key pathobiological feature of diseases like proliferative diabetic retinopathy (PDR), retinopathy of prematurity (ROP), and wet age-related macular

This work was supported by the Retina Research Foundation, the BrightFocus Foundation, the National Institutes of Health National Eye Institute [Grant R01EY025641], and an unrestricted grant from Research to Prevent Blindness, Inc. to T.W.C. Additional financial support for this work was provided by the National Cancer Institute [Grants R01CA138798 (to M.L.F. and M.R.K.), R01CA167291 (M.R.K.), R01CA205166 (M.R.K.)] and the National Institute of Neurologic Disorders and Stroke [Grant R21NS091667 (to M.R.K.)], as well as financial support provided by the Earl and Betty Herr Professor in Pediatric Oncology Research, Jeff Gordon Children's Foundation and the Riley Children's Foundation (M.R.K.). APX3330, APX2009, and APX2014 were provided at no cost by Apexian Pharmaceuticals. Disclosure of potential conflict of interest: M.R.K. has licensed APX3330, APX2009, and APX2014 through Indiana University Research and Technology Corporation to Apexian Pharmaceuticals. M.R.K. and T.W.C. have filed a patent application related to this work. J.H.W. is a consultant chemist for Apexian Pharmaceuticals. Apexian Pharmaceuticals had neither control nor oversight of the studies, interpretation, or presentation of the data in this manuscript. They did not have to approve the manuscript in any way prior to its submission.

${ }^{1}$ S.P.B.S.P. and K.S. contributed equally to this work.

https://doi.org/10.1124/jpet.118.248088.

S This article has supplemental material available at jpet.aspetjournals.org. degeneration (AMD), which together are major causes of blindness (Campochiaro, 2013). In PDR and ROP, abnormal blood vessels grow in and on the retina, and in wet AMD, neovessels grow from the pigmented, subretinal choroid layer into the retina. In all cases, neovessels disrupt retinal architecture and can hemorrhage, leading to blindness. Although the exact stimuli promoting neovascularization are not always well characterized, hypoxia and inflammation both play crucial roles. The currently used, Food and Drug Administrationapproved pharmacological treatments for these diseases are all biologics targeting the vascular endothelial growth factor (VEGF) signaling pathway, such as ranibizumab and aflibercept (Prasad et al., 2010). Although these therapeutic agents have been very successful, significant proportions of patients are resistant and refractory (Lux et al., 2007; Falavarjani and Nguyen, 2013). Moreover, serious side effects including hemorrhage and endophthalmitis are possible. Therefore, development of novel therapeutic approaches targeting other signaling pathways is crucial.

One such potential target is the reduction-oxidation factor 1-apurinic/apyrimidinic endonuclease 1 (Ref-1/APE1), an intracellular signaling nexus with important roles in transducing 
proangiogenic stimuli. This bifunctional protein has an endonuclease role essential for base excision repair (APE1), whereas the Ref- 1 activity is a redox-sensitive transcriptional activator (Shah et al., 2017). Ref-1 redox signaling is a highly regulated process that reduces oxidized cysteine residues in specific transcription factors as part of their transactivation (Xanthoudakis and Curran, 1992; Xanthoudakis et al., 1992; Evans et al., 2000; Lando et al., 2000; Nishi et al., 2002; Seo et al., 2002; Li et al., 2010; Fishel et al., 2011, 2015; Cardoso et al., 2012; Kelley et al., 2012; Luo et al., 2012; Zhang et al., 2013; Logsdon et al., 2016). This redox signaling affects numerous transcription factors including hypoxia-inducible factor $1 \alpha(\mathrm{HIF}-1 \alpha)$, nuclear factor $\kappa$ light-chain-enhancer of activated B cells (NF- $\kappa \mathrm{B})$, and others. The regulation of HIF- $1 \alpha$ and NF- $\kappa \mathrm{B}$ are particularly relevant to angiogenesis and eye diseases (Evans et al., 2000; Nishi et al., 2002; Seo et al., 2002; Fishel et al., 2011, 2015; Cardoso et al., 2012; Logsdon et al., 2016).

That Ref-1 activity can be targeted pharmacologically is exciting. APX3330 (formerly called E3330) is a specific Ref-1/ APE1 redox inhibitor. APX3330 has been extensively characterized as a direct, highly selective inhibitor of Ref-1 redox activity that does not affect the protein's endonuclease activity (Luo et al., 2008, 2012; Fishel et al., 2010, 2015; Su et al., 2011; Cardoso et al., 2012; Zhang et al., 2013). Pharmacologic inhibition of Ref-1 via APX3330 blocks Ref-1 redox activity on $\mathrm{NF}-\kappa \mathrm{B}, \mathrm{HIF}-1 \alpha$, activator protein 1 (AP-1), and signal transducer and activator of transcription 3 (STAT3), decreasing transcription factor binding to DNA in vitro (Fishel et al., 2011; Jedinak et al., 2011; Luo et al., 2012). APX3330 has entered phase I clinical trials for safety and recommended phase II dose in cancer patients (NCT03375086); however, the safety and dose administration of APX3330 have been previously established by Eisai Inc. through a prior development program for a noncancer, hepatitis $\mathrm{C}$ indication that evaluated the preclinical toxicology, phase I and phase II safety, and clinical profile in more than 400 noncancer patients (Shah et al., 2017).

Ref-1/APE1 is highly expressed during retinal development, and in retinal pigment epithelium cells, pericytes, choroidal endothelial cells, and retinal endothelial cells (Chiarini et al., 2000; Jiang et al., 2011; Li et al., 2014a). More generally, Ref-1 is frequently upregulated in regions of tissues in which inflammation is present (Zou et al., 2009; Kelley et al., 2010). APX3330 was previously shown to block in vitro angiogenesis, as evidenced by proliferation, migration, and tube formation of retinal and choroidal endothelial cells (Jiang et al., 2011; Li et al., 2014b). Indeed, APX3330 delivered intravitreally (directly into the eye) reduced neovascularization in the very low density lipoprotein receptor knockout mouse model of retinal neovascularization (Jiang et al., 2011), and also in laser-induced choroidal neovascularization (L-CNV) (Li et al., 2014b), the most widely used animal model that recapitulates features of wet AMD (Grossniklaus et al., 2010).

Although the lead clinical candidate is effective in preclinical cancer studies, we also sought novel, second generation Ref-1 inhibitors that would increase efficacy in antiangiogenic and anti-inflammatory transcription factor (NF- $\kappa \mathrm{B}, \mathrm{HIF}-1 \alpha)$ inhibition, as well as new chemical properties. We present here the synthesis of APX3330 derivatives APX2009 [reported previously as a neuroprotective agent (Kelley et al., 2016)], and APX2014 [reported here for the first time (Kelley and Wikel, 2015)]. We go on to show that these compounds have antiangiogenic activity against retinal and choroidal endothelial cells both in culture and in an ex vivo choroidal sprouting model. Finally, we show that APX2009 is an effective systemic treatment for L-CNV, establishing these compounds as leads for further development for neovascular eye diseases.

\section{Materials and Methods}

Synthetic Methods. The compounds were synthesized by Cascade Custom Chemistry (Eugene, OR) and provided by Apexian Pharmaceuticals, Indianapolis, IN. In summary (Fig. 1A), the common intermediate iodolawsone (2-iodo-3-hydroxy-1,4-naphthoquinone (1); available from Cascade Custom Chemistry) was treated with 2-propylacrylic acid (2), with oxalyl chloride and the corresponding amine, and with sodium methoxide in methanol to yield $(E)-N, N$-diethyl-2-((3-methoxy-1,4dioxo-1,4-dihydronaphthalen-2-yl)methylene)pentanamide (6a; APX2009), and (E)- $N$-methoxy-2-((3-methoxy-1,4-dioxo-1,4-dihydronaphthalen-2-yl)methylene)pentanamide (6b; APX2014). Full synthetic details can be found in the Supplemental Methods. APX3330 was synthesized as described (Luo et al., 2008).

Electrophoretic Mobility Shift Assays. These assays were performed as previously described (Luo et al., 2008, 2012; Kelley et al., 2011; Su et al., 2011; Zhang et al., 2013). Briefly, an increasing amount of APX3330, APX2009, or APX2014 was preincubated with purified Ref-1 protein in electrophoretic mobility shift (EMSA) reaction buffer for 30 minutes. The EMSA assay was performed using the AP-1 target DNA sequence and AP-1 protein.

Cells. Primary human retinal microvascular endothelial cells (HRECs) were obtained from Cell Systems, Inc. (Kirkland, WA), and the Rf/6a macaque choroidal endothelial cell line was obtained from American Type Culture Collection (Manassas, VA). Cells were maintained as described (Basavarajappa et al., 2017), reordered at least annually, and regularly assessed for mycoplasma contamination.

In Vitro Cell Proliferation Assay. Endothelial cell proliferation was assessed as described previously (Basavarajappa et al., 2014, 2017). Briefly, $2.5 \times 10^{3}$ cells were seeded in $100 \mu$ l of growth medium and plated in each well of 96 -well clear-bottomed black plates and incubated for 24 hours. APX2009, APX2014, or dimethyl sulfoxide (DMSO) vehicle (DMSO final concentration $=1 \%$ ) was added, and the plates were incubated for $24-48$ hours at $37^{\circ} \mathrm{C}$ and $5 \% \mathrm{CO}_{2}$. alamarBlue reagent $(11.1 \mu \mathrm{l})$ was added to each well of the plate and 4 hours later fluorescence readings were taken at excitation and emission wavelengths of 560 and $590 \mathrm{~nm}$, respectively, using a Synergy H1 plate reader (BioTek, Winooski, VT). Median growth inhibitory concentration $\left(\mathrm{GI}_{50}\right)$ was calculated using GraphPad Prism v. 7.0.

\footnotetext{
ABBREVIATIONS: AMD, age-related macular degeneration; ANOVA, analysis of variance; AP-1, activator protein 1; APE1, apurinic/apyrimidinic endonuclease 1; APX2009, (E)-N,N-diethyl-2-((3-methoxy-1,4-dioxo-1,4-dihydronaphthalen-2-yl)methylene)pentanamide; APX2014, (E)- $N$ methoxy-2-((3-methoxy-1,4-dioxo-1,4-dihydronaphthalen-2-yl)methylene)pentanamide; APX3330, (2E)-2-[(4,5-dimethoxy-2-methyl-3,6-dioxo-1,4cyclohexadien-1-yl)methylene]-undecanoic acid; DAPI, 4',6-diamidine-2'-phenylindole; DMSO, dimethyl sulfoxide; EBM-2, endothelial basal medium 2; EdU, 5-ethynyl-2'-deoxyuridine; EGM-2, endothelial growth medium 2; EMSA, electrophoretic mobility shift assay; $\mathrm{Gl}_{50}$, median growth inhibitory concentration; HIF- $1 \alpha$, hypoxia-inducible factor $1 \alpha$; HRECs, human retinal microvascular endothelial cells; L-CNV, laser-induced choroidal neovascularization; NF- $\kappa$ B, nuclear factor $\kappa$ light-chain-enhancer of activated B cells; OCT, optical coherence tomography; PBS, phosphate-buffered saline; PDR, proliferative diabetic retinopathy; Ref-1, reduction-oxidation factor 1; ROP, retinopathy of prematurity; TNF, tumor necrosis factor; TUNEL, terminal deoxynucleotidyl transferase dUTP nick end labeling; VEGF, vascular endothelial growth factor.
} 
A<smiles>O=C1C(O)=C(I)C(=O)c2ccccc21</smiles>

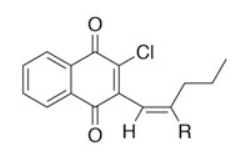

5a, R= $=\mathrm{CONEt}_{2}$

5b, R= $=\mathrm{CONHOCH}_{3}$

$\downarrow d$

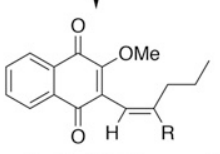

6a, R=CONEt $\quad$ APX2009

$\begin{array}{ll}6 \mathrm{a}, \mathrm{R}=\mathrm{CONEt}_{2} & \text { APX2009 } \\ 6 \mathrm{~b}, \mathrm{R}=\mathrm{CONHOCH}_{3} & \text { APX2014 }\end{array}$
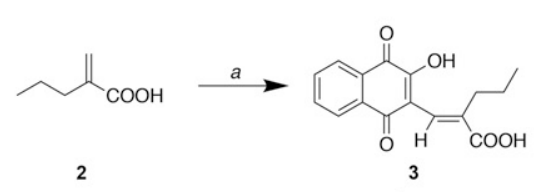

(c)

4

B

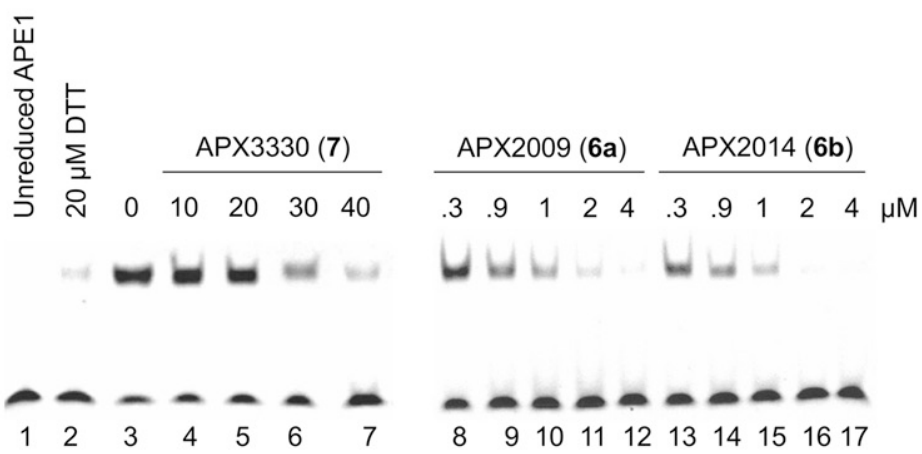

Fig. 1. Synthesis and activity of Ref-1 inhibitors. (A) Synthetic scheme for APX2009 (6a) and APX2014 (6b). Structure of APX3330 (7) included for reference. Reagents and conditions: $a, 2$-iodo-3-hydroxy-1,4-naphthoquinone (iodolawsone, 1), 2-propylacrylic acid $(\mathbf{2}), \mathrm{K}_{2} \mathrm{CO}_{3}, \mathrm{Pd}(\mathrm{OAc})_{2}, \operatorname{argon}_{1}, 100^{\circ} \mathrm{C}$, 1 hour, $74 \% ; b,(\mathrm{COCl})_{2}$, DMF, DCM, RT overnight, $100 \% ; c$, DEA·HCl (APX2009) or $\mathrm{CH}_{3} \mathrm{ONH}_{2} \cdot \mathrm{HCl}(\mathrm{APX} 2014), \mathrm{DIPA} \cdot \mathrm{HCl}, \mathrm{RT}, 45 \mathrm{minutes}, 62 \%$ and $71 \%$ respectively; $d, \mathrm{NaOCH}_{3} / \mathrm{CH}_{3} \mathrm{OH}$, argon, 30 minutes, RT, $96 \%$ and $86 \%$, respectively. See Supplemental Methods for full synthetic details. (B) APX2009 and APX2014 are more effective inhibitors of Ref-1-induced AP-1 DNA binding than APX3330 in an EMSA. Two separate gels from the same experiment are shown. The $\mathrm{IC}_{50}$ for redox EMSA inhibition was $25,0.45$, and $0.2 \mu \mathrm{M}$ for APX3330, APX2009, and APX2014, respectively. These assays were performed multiple times with similar results.

EdU Incorporation, Ki-67 Staining, and Terminal Deoxynucleotidyl Transferase dUTP Nick End Labeling. These assays were carried out as described previously (Basavarajappa et al., 2014, 2017) but using chamber slides not cover slips. Briefly, cells $(30,000$ per well) were seeded on eight-well chamber slides coated with attachment factors and allowed to attach overnight. Cells were treated with the indicated compound concentrations for 17 hours (overnight). To assay proliferation, cells were incubated with EdU in complete media for 8 hours at $37^{\circ} \mathrm{C}$. Cells were then fixed in $4 \%$ paraformaldehyde for 20 minutes and permeabilized using $0.25 \%$ Triton X-100 prepared in phosphate-buffered saline (PBS). Cells were incubated with a rabbit-specific monoclonal antibody against Ki-67 (D3B5) (cat. no. 9129; Cell Signaling Technologies, Danvers, MA) (1:400) overnight at $4^{\circ} \mathrm{C}$. Secondary antibody was Alexa Fluor goat anti-rabbit 488 (A11034; Invitrogen/Thermo Fisher Scientific, Carlsbad, CA) with DAPI counterstain for nuclear staining. Proliferating cells that incorporated EdU were detected using the Click-iT EdU imaging kit (Invitrogen). Alternatively, apoptotic cells were visualized using the Click-iT TUNEL assay kit (Invitrogen) per the manufacturer's instructions, with Hoechst 33342 counterstain for nuclear staining, and a 17-hour treatment with $1 \mu \mathrm{M}$ staurosporine as positive control. The cells were imaged using a Zeiss AxioImager D2 microscope or an LSM 700 confocal microscope and the percentage of positive cells was counted on three low-power (for TUNEL) or high-power (for Ki-67 and EdU) fields per well using ImageJ software.

Cell Cycle Analysis. HRECs $\left(2 \times 10^{6}\right)$ were grown in endothelial growth medium 2 (EGM-2). Cells were serum-starved in endothelial basal medium 2 (EBM-2) medium overnight, then treated with the indicated concentrations of APX2009 or APX2014 along with DMSO control for 24 hours in complete medium. Cells were washed twice in ice-cold PBS followed by fixation in $66 \%$ ethanol solution overnight at $4^{\circ} \mathrm{C}$. Fixed cells were again washed twice in ice-cold PBS and the pellets were resuspended in propidium iodide staining solution for 30 minutes at $37^{\circ} \mathrm{C}(20 \mu \mathrm{g} / \mathrm{ml}$ propidium iodide prepared in $1 \times$ PBS containing $0.1 \%$ Triton X-100 and $100 \mu \mathrm{g} / \mathrm{ml}$ RNase A). After incubation cells were analyzed using flow cytometry (FACSCalibur; BD Biosciences, San Jose, CA). Pulse shape analysis was used to exclude doublets and debris. The single-cell population was then assessed by the FL2 area histogram plot using ModFit software (v. 5.0) and cell cycle profiles were generated.

In Vitro Cell Migration Assay. Endothelial cell migration was monitored as described before (Basavarajappa et al., 2014, 2017). Briefly, HRECs and Rf/6a were grown until confluency in 12 -well plates. Using a sterile $10-\mu l$ micropipette tip, a scratch wound was made across the center of each well and fresh complete media containing DMSO or different concentrations of APX2009 or APX2014 compounds were added to the wells (DMSO final concentration $=1 \%$ ). Wells were imaged via digital bright-field microscopy at different time points, and the number of migrated cells into the scratched area was manually counted.

In Vitro Matrigel Tube Formation Assay. The ability of HRECs and $\mathrm{Rf} / 6$ a cells to form tubes in vitro was monitored as described before (Basavarajappa et al., 2014, 2017). Briefly, $1.5 \times 10^{4}$ cells in $100 \mu \mathrm{l}$ of growth medium containing DMSO or APX compounds were added to each well of a 96 -well plate that was precoated with $50 \mu \mathrm{l}$ per well of Matrigel basement membrane (DMSO final concentration $=1 \%$ ). Bright-field digital micrographs of each well at different time points were taken to measure the in vitro tube formation using the Angiogenesis Analyzer plugin in ImageJ software (v.1.48; http://image.bio.methods.free.fr/ImageJ/?AngiogenesisAnalyzer-for-ImageJ.html).

NF-кB p65 Nuclear Translocation Assay. The NF- $\kappa$ B nuclear translocation assay was performed by seeding 30,000 HRECs/well on an eight-well chamber slide coated with attachment factors. The cells were grown in EGM-2 medium overnight before treatment with indicated concentrations of APX2009 and APX2014, or $10 \mu \mathrm{M}$ BAY 11-7082 (MilliporeSigma, St. Louis, MO) as a positive control NF- $\kappa$ B inhibitor. After a 17-hour incubation, the medium was replaced with EBM-2 (minimal medium) with indicated concentrations of compound or DMSO for 1 hour. The cells were then stimulated with $10 \mathrm{ng} / \mathrm{ml}$ tumor necrosis factor (TNF)- $\alpha$ in EBM-2 for 20 minutes at $37^{\circ} \mathrm{C}$ to activate NF- $\kappa$ B. Cells were then fixed in $4 \%$ paraformaldehyde and permeabilized using $0.5 \%$ Triton X-100 solution prepared in PBS. The cells were incubated with a monoclonal antibody against NF- $\kappa$ B p65 (sc-8008; Santa Cruz Biotechnology, Dallas, TX) (1:50) overnight at $4^{\circ} \mathrm{C}$, followed by Alexa Fluor 555 goat anti-mouse secondary antibody (1:2000) for 1 hour. The cells were counterstained with Hoechst 33342 for nuclear staining and then mounted using Everbrite hardset 
A

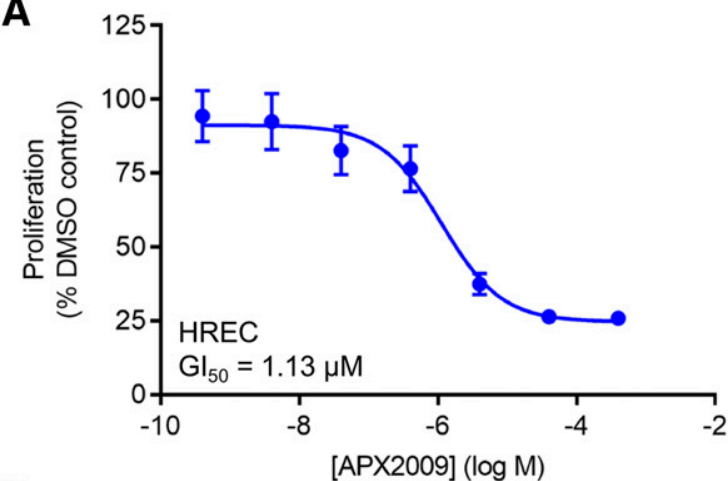

B

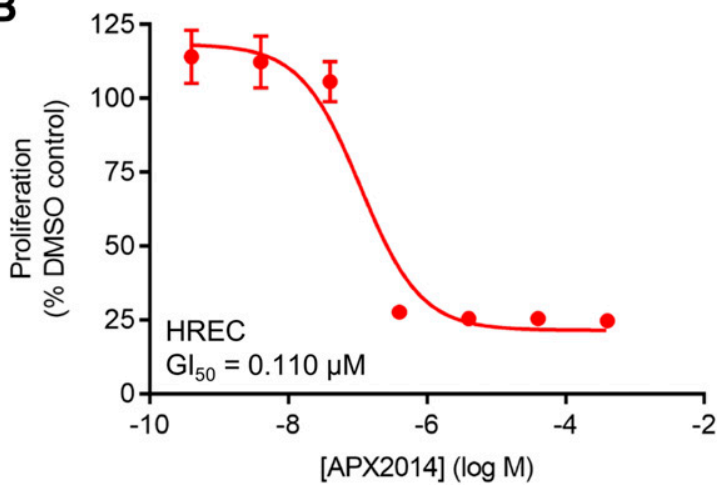

C
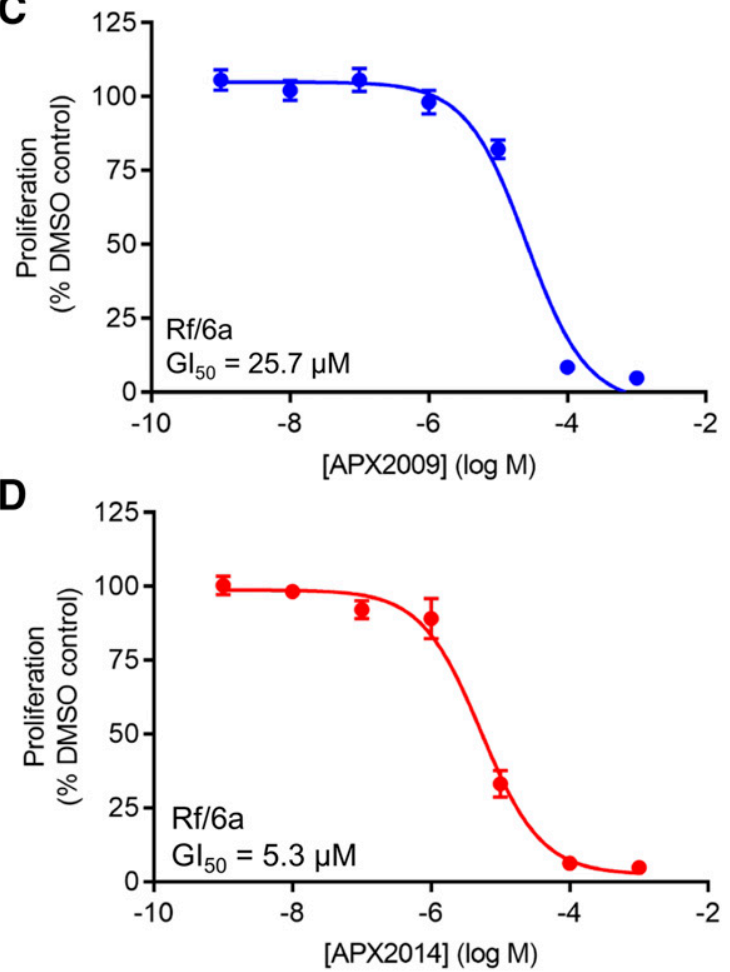

Fig. 2. Compounds APX2009 and APX2014 inhibit endothelial cell proliferation in HRECs and Rf/6a cells in vitro. Dose-dependent effects of APX2009 (A) and APX2014 (B) in HRECs, and dose-dependent effects of APX2009 (C) and APX2014 (D) in Rf/6a choroidal endothelial cells. In vitro proliferation was measured using an alamarBlue assay. $\mathrm{GI}_{50}$ values are indicated. Mean \pm S.E.M., $n=3$ per dose.

mounting medium. The cells were imaged using a Zeiss AxioImager D2 microscope.

Quantitative Reverse Transcription-Polymerase Chain Reaction. The assay was performed as described previously (Basavarajappa et al., 2014, 2017). RNA was extracted from cells treated as indicated using Trizol (Invitrogen). cDNA was synthesized from $1 \mu \mathrm{g}$ of RNA using random primers and iScript reverse transcriptase (Bio-Rad, Hercules, CA). Quantitative reverse transcription-polymerase chain reaction (qPCR) was performed in $10 \mu \mathrm{l}$ volumes in a 384-well plate, with Fast Advanced Master Mix and TaqMan probes on a ViiA7 thermal cycler (Applied Biosystems, Foster City, CA). Primer/probesets used were as follows: VEGFA (Hs00900055_m1), VCAM1 (Hs01003372_m1), and CCL2O
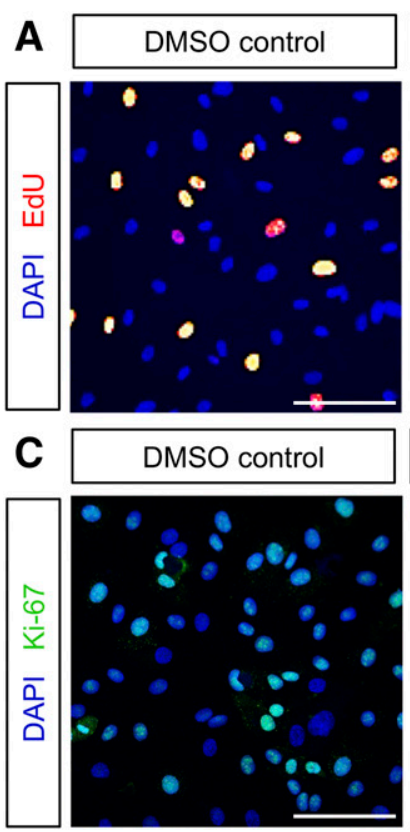
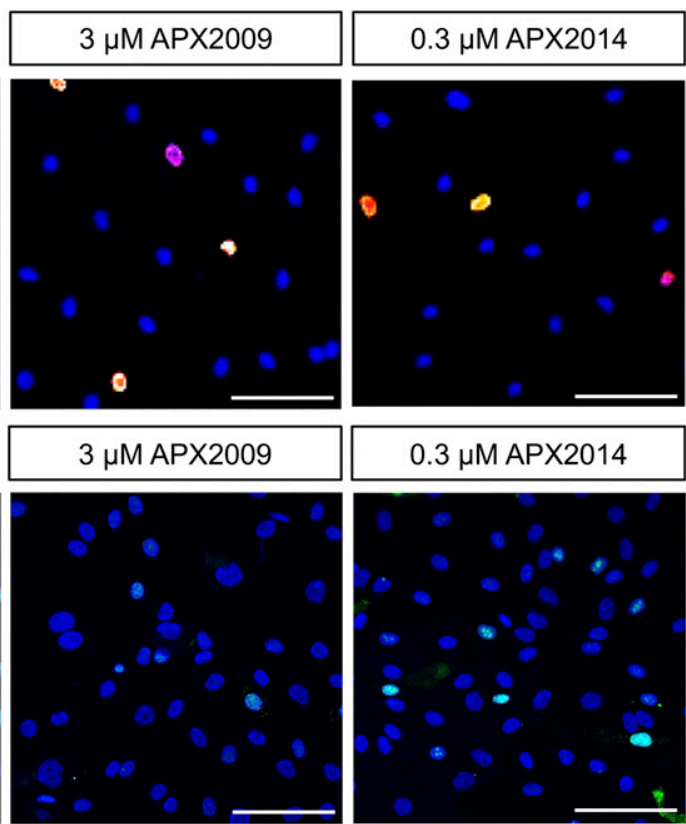
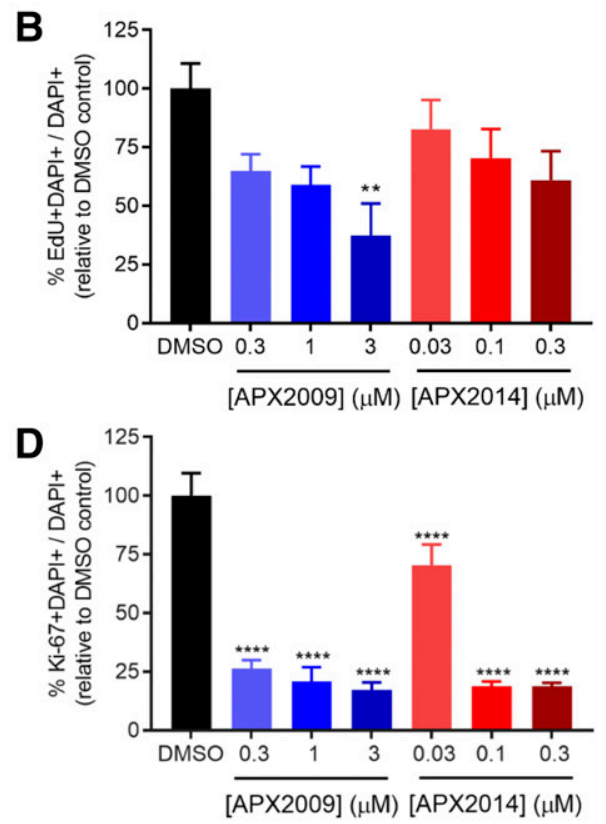

Fig. 3. Compounds APX2009 and APX2014 inhibit S phase in HRECs. After HRECs were treated with the indicated concentrations of APX2009 and APX2014, (A) EdU (red) and (C) Ki-67 (green) were detected and nuclei (blue) stained with DAPI; Scale bars, $100 \mu \mathrm{m}$. (B) Quantification of EdU and (D) quantification of Ki-67 in HRECs. Mean \pm S.E.M., $n=3$ fields per dose. ${ }^{* *} P<0.01$; $* * * * P<0.0001$ compared with DMSO control (one-way ANOVA with Dunnett's post-hoc test). Representative data from three independent experiments. See Supplemental Figs. $1 \mathrm{~A}$ and 2. 
(Hs01011368_m1), and housekeeping controls HPRT (Hs02800695_m1) and TBP (Hs00427620_m1). The data were analyzed using the $\Delta \Delta \mathrm{C}_{\mathrm{t}}$ method. The expression levels of genes were normalized to the two housekeeping genes and calibrated to the DMSO-treated sample.

Animals. All animal experiments were approved by the Indiana University School of Medicine Institutional Animal Care and Use Committee and followed the guidelines of the Association for Research in Vision and Ophthalmology Statement for the Use of Animals in Ophthalmic and Visual Research. Wild-type female C57BL/6 mice, 6-8 weeks of age, were purchased from Envigo (Indianapolis, IN; for choroidal sprouting experiments) or Jackson Laboratory (Bar Harbor, $\mathrm{ME}$; for L-CNV) and housed under standard conditions (Wenzel et al., 2015). Mice were anesthetized for all procedures by intraperitoneal injections of $90 \mathrm{mg} / \mathrm{kg}$ ketamine hydrochloride and $5 \mathrm{mg} / \mathrm{kg}$ xylazine, with intraperitoneal atipamezole reversal $(1 \mathrm{mg} / \mathrm{kg})$. Treatments were randomly assigned by cage.

Choroidal Sprouting Assay. Ex vivo choroidal sprouting was assessed as described previously (Sulaiman et al., 2016; Basavarajappa et al., 2017). Briefly, choroid-sclera was dissected from 7- to 8-week old mouse eyes and pieces were embedded in Matrigel (growth factor reduced) and grown in EGM-2 containing antibiotics for 72 hours to allow sprouting to initiate. The indicated concentrations of APX2009 and APX2014 compounds (in DMSO, final DMSO concentration 0.5\% and $0.2 \%$, respectively) were added and growth allowed to proceed for 48 hours. Images were taken and growth was quantified by measuring the distance from the edge of the choroidal piece to the growth front in four directions per sample using ImageJ software.

Laser-Induced Choroidal Neovascularization. L-CNV was induced as described previously (Sulaiman et al., 2015, 2016; Basavarajappa et al., 2017). Studies were powered to have an $80 \%$ chance of detecting effect size-differences of 50\%, assuming 30\% variability, $\alpha=0.05$. Briefly, pupils of anesthetized mice were dilated with 1\% tropicamide (Alcon Laboratories Inc., Forth Worth, TX) and lubricated with hypromellose ophthalmic demulcent solution (Gonak; Akorn, Lake Forest, IL). A cover slip was used to allow viewing of the posterior pole of the eye. Three burns of a 532-nm ophthalmic argon green laser coupled with a slit lamp (50- $\mu \mathrm{m}$ spot size, 50-millisecond duration, and $250-\mathrm{mW}$ pulses) were delivered to each 3,9 , and 12 o'clock position, two-disc diameters from the optic disc. The bubbling or pop sensed after laser photocoagulation was considered

\section{C}
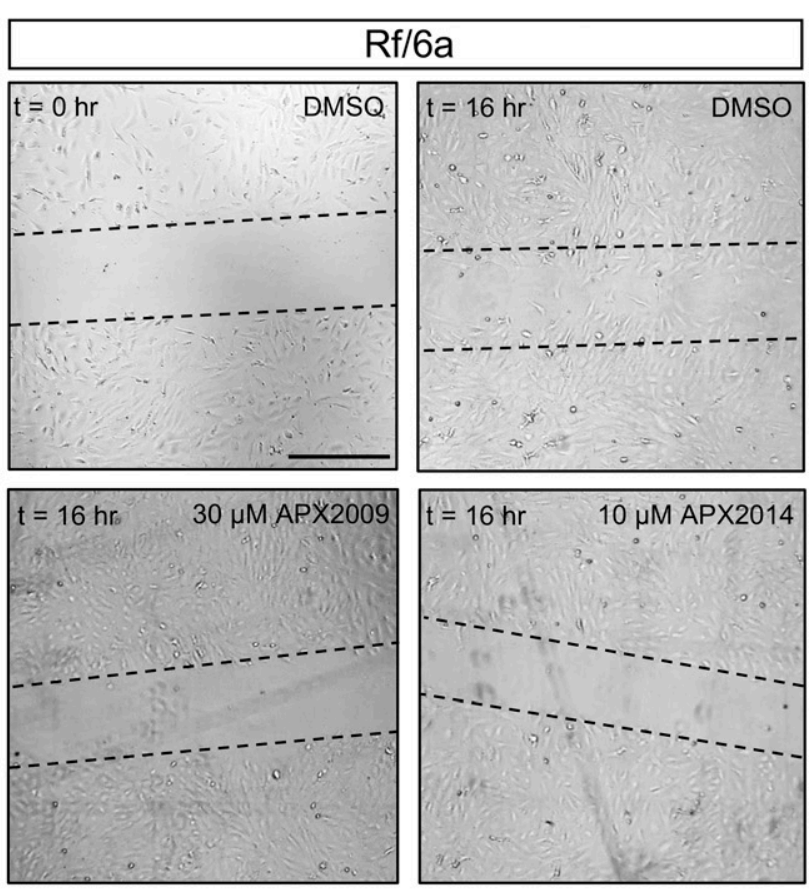

D

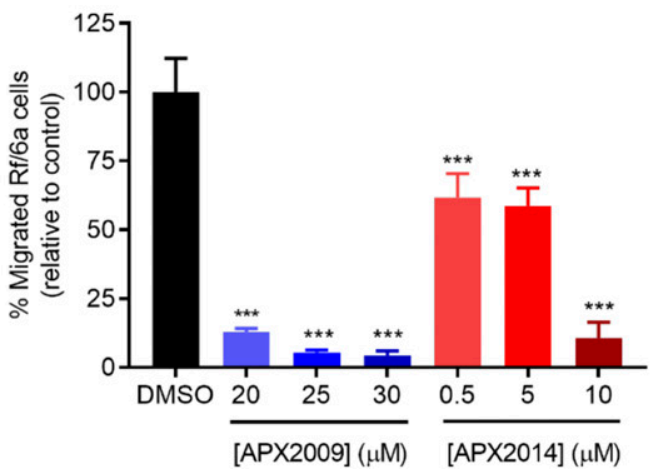

Fig. 4. Compounds APX2009 and APX2014 inhibit endothelial cell migration in HRECs and Rf/6a cells in vitro. (A) Effect of APX2009 and APX2014 on cell migration in HRECs. A confluent monolayer of HRECs with various treatments (highest doses shown) was wounded and wound closure was monitored for 8 hours. (B) Quantitative analysis of cell migration shows that APX compounds significantly block the migration of HRECs. (C) Effect of APX2009 and APX2014 on cell migration in Rf/6a cells. A confluent monolayer of Rf/6a with various treatments (highest doses shown) was wounded and wound closure was monitored for 16 hours. (D) Quantitative analysis of cell migration shows that APX compounds significantly block the migration of $\mathrm{Rf} / 6$ a cells. Mean \pm S.E.M., $n=3$ per dose. ${ }^{*} P<0.01 ; * * P<0.001$ compared with DMSO control (one-way ANOVA with Dunnett's post-hoc test). Representative data from three independent experiments. Scale bar, $500 \mu \mathrm{m}$. See Supplemental Figs. 4 and 5. 
A

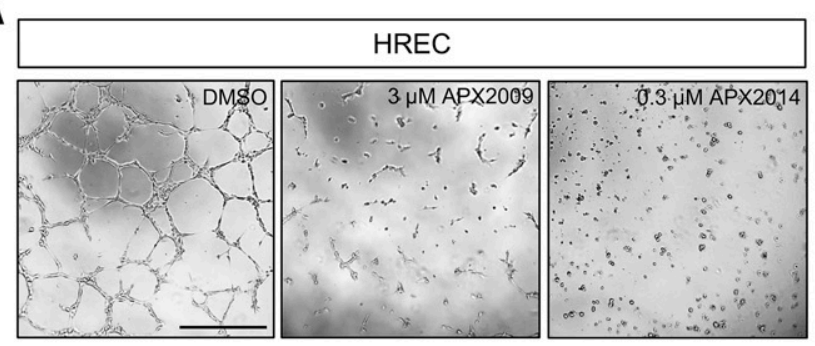

B

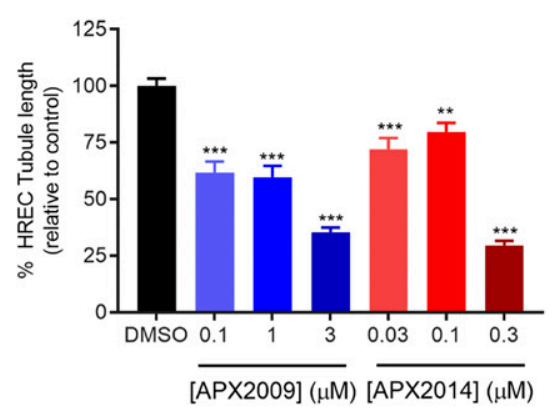

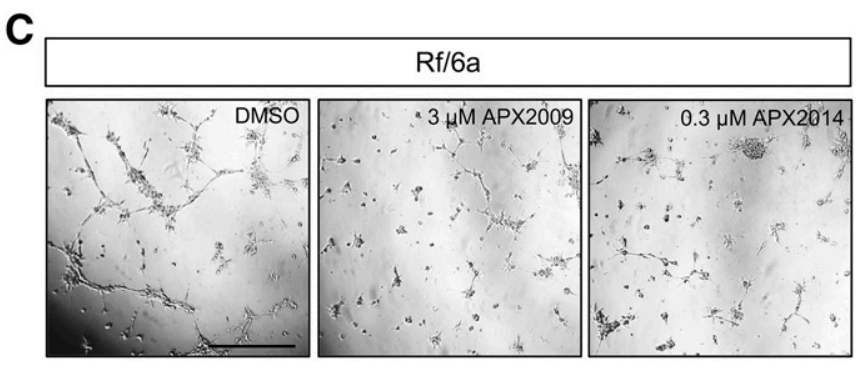

D

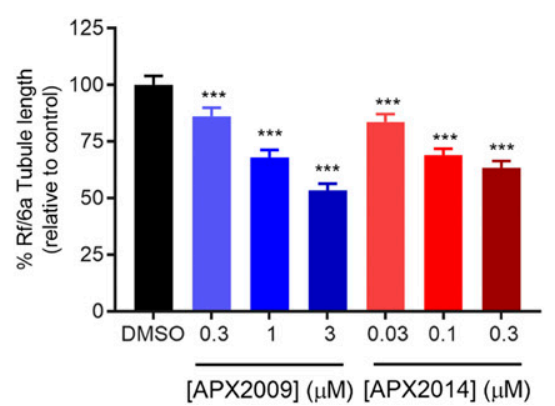

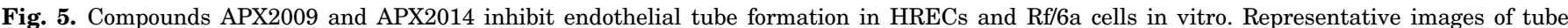

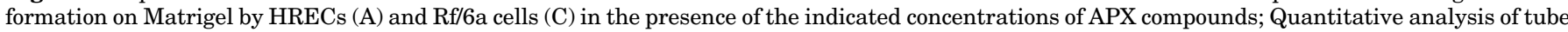

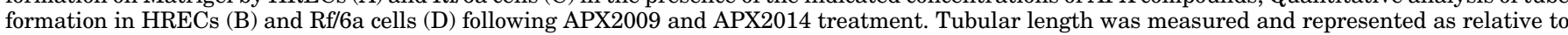

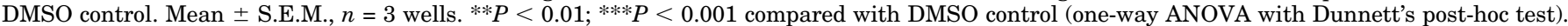
Representative data from three independent experiments. Scale bar, $500 \mu \mathrm{m}$. See Supplemental Figs. 6 and 7.

as the successful rupture of Bruch's membrane. Lesions in which bubbles were not observed were excluded from the study. To assess the antiangiogenic activity of APX3330, the mice were injected intraperitoneally with compound ( $50 \mathrm{mg} / \mathrm{kg}$ body weight), twice daily, 5 days on/2 days off, as used previously in vivo (Fishel et al., 2011; Lou et al., 2014; Biswas et al., 2015). Vehicle was 4\% Cremophor/ethanol (1:1) in PBS. For APX2009, doses were 12.5 or $25 \mathrm{mg} / \mathrm{kg}$ body weight, twice daily until 14 days of laser treatment unless otherwise indicated. Vehicle was propylene glycol, Kolliphor HS15, Tween 80 (McIlwain et al., 2017). Mice were weighed daily.

In Vivo Imaging. Optical coherence tomography (OCT) was performed in L-CNV mice as described previously (Sulaiman et al., 2016) at the indicated times using the Micron III intraocular imaging system (Phoenix Research Laboratories, Pleasanton, CA). Briefly, before the procedure, eyes of anesthetized mice were dilated with $1 \%$ tropicamide solution (Alcon) and lubricated with hypromellose ophthalmic demulcent solution (Gonak; Akorn). Mice were then placed on a custom heated stage that moved freely to position the mouse eye for imaging. Several horizontal and vertical OCT images were taken per lesion. Fluorescein angiography was performed 14 days post-laser by intraperitoneal injection of $50 \mu \mathrm{l}$ of $25 \%$ fluorescein sodium (Fisher Scientific, Pittsburgh, PA). Fundus images were taken using the Micron III system and StreamPix software (Nor-Pix, Montreal, Quebec, Canada).

Choroidal Flatmount Immunofluorescence. Mouse eyes were harvested 14 days after L-CNV induction. The eyes were enucleated and fixed in $4 \%$ paraformaldehyde/PBS overnight. The anterior segment, lens, and retina were removed, and the posterior eye cups were prepared for choroidal flat mounts. The posterior eye cups were washed with PBS and permeabilized in blocking buffer containing $0.3 \%$ Triton X-100 and 5\% bovine serum albumin (BSA) in PBS for 2 hours at $4^{\circ} \mathrm{C}$. The eye cups were then double stained for vasculature with rhodamine-labeled Ricinus communis agglutinin I (Vector Laboratories, Burlingame, CA) and Alexa Fluor 488-conjugatedIsolectin B4 from Griffonia simplicifolia (GS-IB4) (Molecular Probes/ Thermo Fisher Scientific) at 1:250 dilution in buffer containing 0.3\% Triton X-100, $0.5 \%$ BSA in PBS for $16-20$ hours at $4^{\circ} \mathrm{C}$. After antibody incubation, whole mounts were washed three times with PBS for 15 minutes, each step at $4^{\circ} \mathrm{C}$ with $0.1 \%$ Triton X-100. After washing, choroidal flatmounts were mounted in aqueous mounting medium (VectaShield; Vector Laboratories, Inc.) and cover-slipped for observation by confocal $Z$-stack imaging (LSM 700; Zeiss, Thornwood, NY) to estimate lesion volume. The sum of the stained area in each optical section, multiplied by the distance between sections $(3 \mu \mathrm{m})$, gave the choroidal neovascularization lesion volume and lesion volume was quantified using ImageJ software. Lesions were only included for analysis if they met quality control standards as published (Poor et al., 2014). All lesions in an eye were averaged to represent a single $n$.

Statistical Analyses. Statistical analyses were performed with GraphPad Prism 7 software. One-way ANOVA was used with Dunnett's post-hoc test for EdU, Ki-67, TUNEL, cell cycle, migration, tube formation, qPCR, and choroidal sprouting experiments. Unpaired Student's $t$ test was used for the APX3330 in vivo experiment. One-way ANOVA was used with Tukey's post-hoc test for L-CNV analysis in APX2009 in vivo experiments, and repeated-measures two-way ANOVA was used to compare body weights between treatments and over time. Two-sided $P$ values $<0.05$ were considered statistically significant.

\section{Results}

Novel Ref-1 Inhibitors Are More Potent than APX3330 in Blocking Binding of AP-1 to DNA. We synthesized APX2009 (6a) and APX2014 (6b) (Fig. 1A) and demonstrated that both compounds had enhanced inhibition of Ref-1-induced transcription factor binding to DNA compared with APX3330 (7) (Fig. 1B), although they have substantially different physiochemical properties. The new compounds have lower molecular weights and lack the carboxylate group and long alkyl chain of APX3330. The new compounds also have significantly reduced lipophilicity as determined by computer-based calculation of their cLogP values, APX3330 $=4.5, \mathrm{APX} 2009=2.7$, and APX2014 $=1.9$.

APX2009 and APX2014 Block Endothelial Cell Proliferation. Endothelial cell proliferation with increased survival supports the cells that make up new blood 

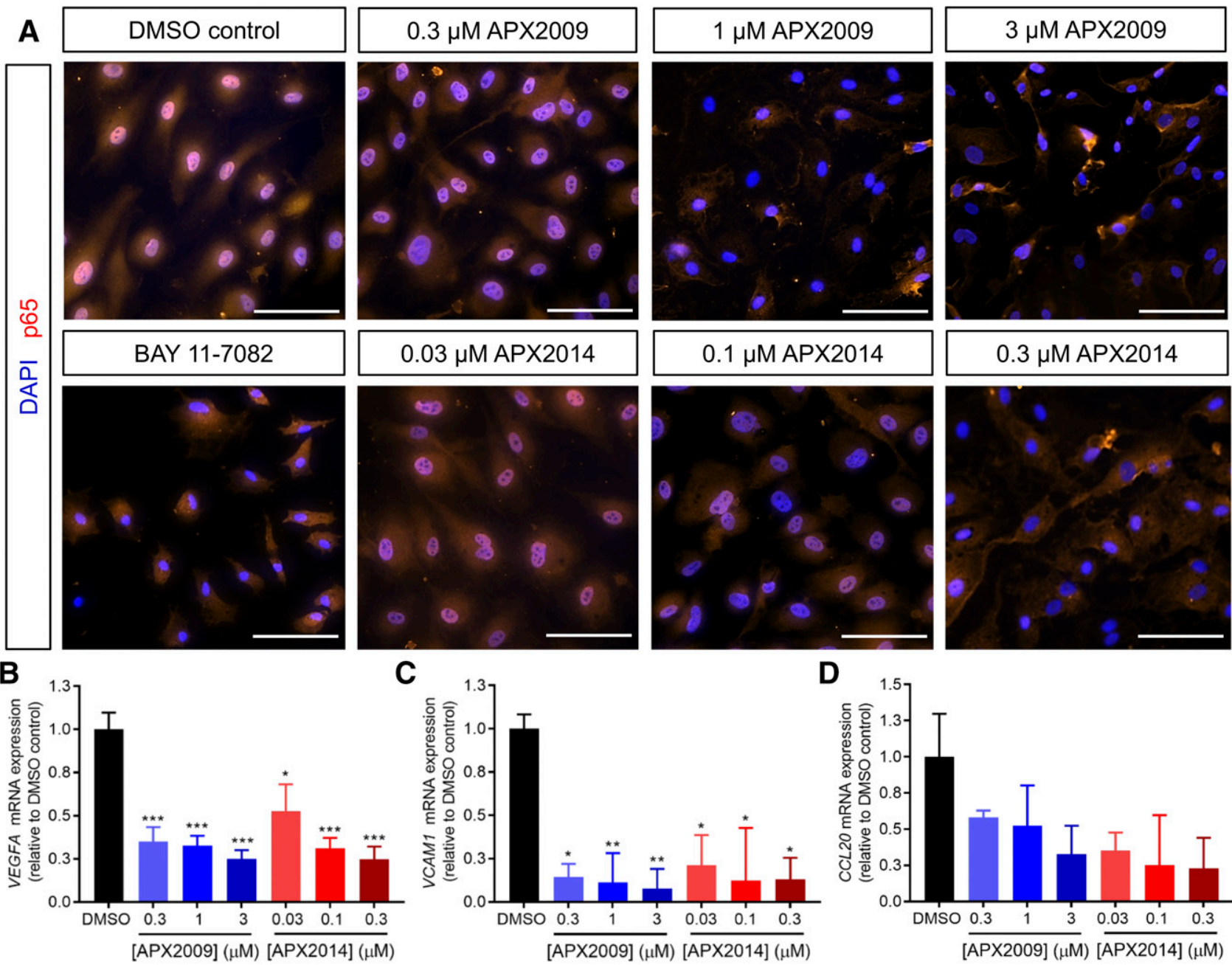

D

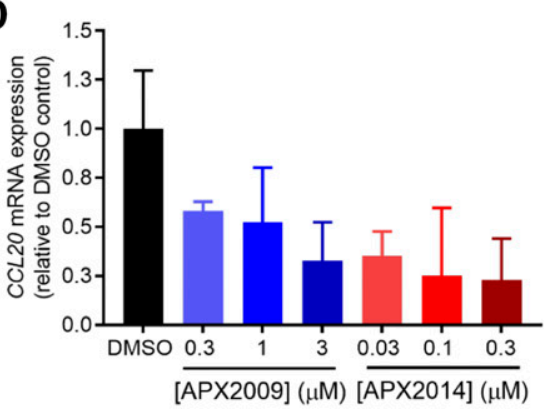

Fig. 6. Compounds APX2009 and APX2014 inhibit TNF- $\alpha$-mediated NF- $\kappa$ B signaling and proangiogenic target gene mRNA expression. (A) After treating HRECs with the indicated concentrations of APX2009 and APX2014, p65 (red) was detected by immunofluorescence and nuclei (blue) stained with DAPI; compounds dose dependently reduced p65 nuclear translocation as evidenced by decreased overlap between red and blue signals. BAY 11-7082 is a positive control NF- $\kappa$ B inhibitor. Scale bars, $100 \mu \mathrm{m}$. (B) VEGFA, (C) VCAM1, and (D) CCL20 mRNA expression levels in HRECs. APX2009 and APX2014 dose dependently inhibited levels of each transcript. Mean \pm S.E.M., $n=3$ technical replicates. ${ }^{*} P<0.05 ; * * P<0.01 ; * * * P<0.001$ compared with DMSO control (one-way ANOVA with Dunnett's post-hoc test). Representative data from three independent experiments.

vessels, leading to angiogenesis. As an initial test of the antiangiogenic potential of our novel Ref-1 inhibitors, we assessed their ability to inhibit the proliferation of HRECs and Rf/6a choroidal endothelial cells (Fig. 2). Both compounds dose dependently blocked proliferation of both cell types in an alamarBlue assay, with APX2014 5- to 10-fold more potent than APX2009. Primary HRECs were more sensitive to both compounds than the Rf/6a choroidal cell line, as seen with other antiangiogenic compounds (Basavarajappa et al., 2017).

APX2009 and APX2014 Block S Phase without Inducing Apoptosis. We assessed the activity of our novel compounds in more detail in HRECs. Both compounds reduced the number of cells going through $\mathrm{S}$ phase as evidenced by reduced Ki-67 staining and reduced EdU incorporation (Fig. 3; Supplemental Figs. 1A and 2). This was also evident as a modest increase in cells in $\mathrm{G}_{0} / \mathrm{G}_{1}$ phase at high doses of compound, with a concomitant decrease in $\mathrm{G}_{2} / \mathrm{M}$ phase cells (Supplemental Fig. 1, B and C). However, neither compound induced apoptosis at antiproliferative doses as assessed by TUNEL (Supplemental Fig. 3).
APX2009 and APX2014 Block Endothelial Cell Migration. Neovascularization involves an array of coordinated events, including extracellular matrix degradation, cell migration, cell proliferation, and morphogenesis of endothelial cells. To know the effect of APX2009 and APX2014 compounds on endothelial cell migration, a scratch-wound assay was performed (Fig. 4; Supplemental Figs. 4 and 5). Both compounds again were dose dependently and significantly effective here, without causing obvious cytotoxicity over the short time-course of these assays.

APX2009 and APX2014 Block Endothelial Cell Tube Formation. Endothelial cells organize and form capillarylike structures upon plating on an extracellular matrix such as Matrigel. The organization of endothelial cells into a threedimensional network of tubes is essential for angiogenesis. As such, the Matrigel tube formation assay is a good in vitro predictor of angiogenic potential in vivo. In this assay, both APX2009 and APX2014 inhibited tubule formation markedly, at concentrations lower than those required for inhibiting migration alone, strongly indicative of antiangiogenic activity (Fig. 5; Supplemental Figs. 6 and 7). 
A
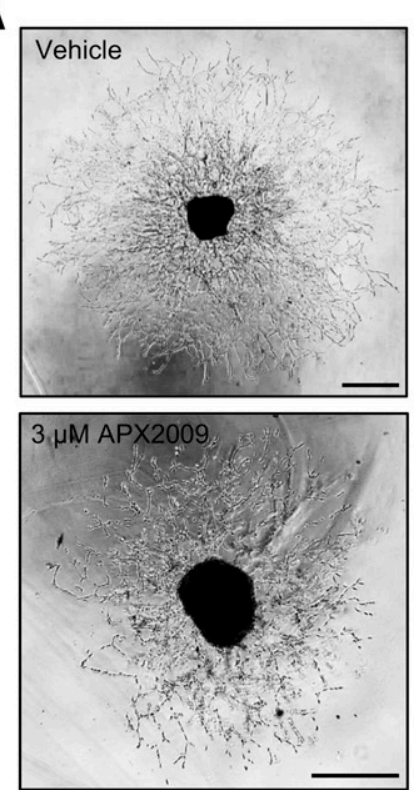

B

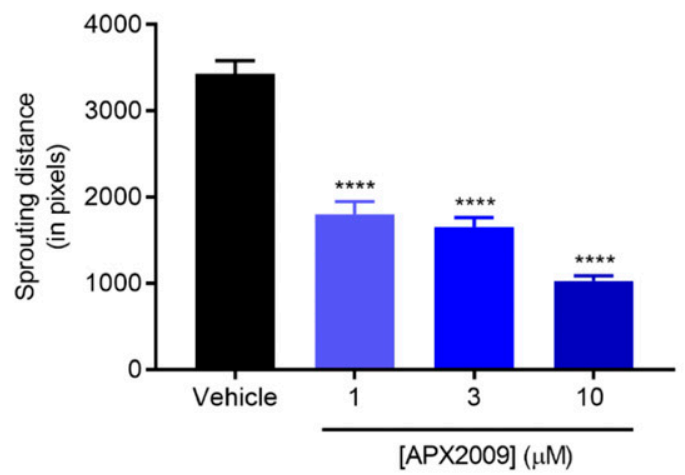

C
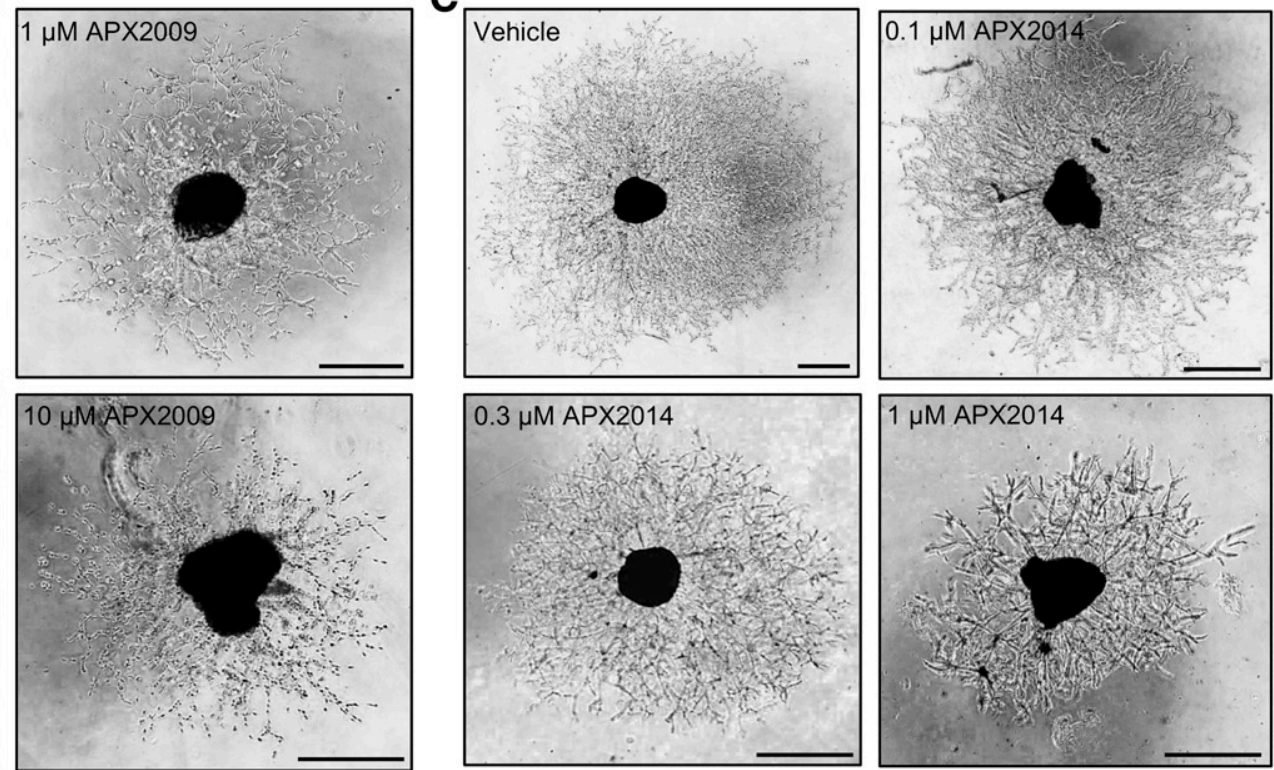

D

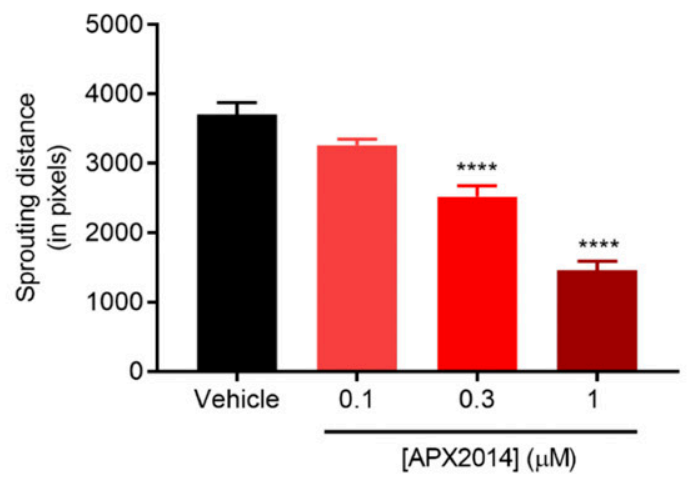

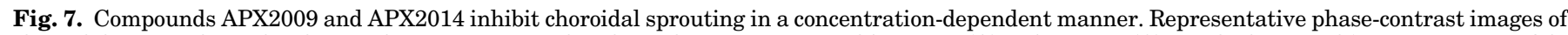

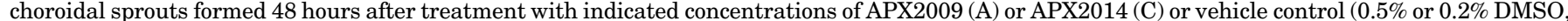

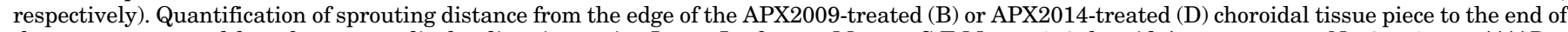

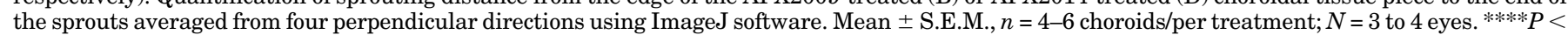
0.0001 (ANOVA with Dunnett's post-hoc test). Scale bars, $500 \mu \mathrm{m}$.

APX2009 and APX2014 Inhibit NF-кB Activity. Since Ref-1 inhibition has previously been associated with reduction in NF- $\kappa$ B activity (Shah et al., 2017), we assessed activity of this pathway in response to our novel compounds in HRECs, to determine if APX2009 and APX2014 were acting through the expected mechanisms. First, we assayed translocation of the $\mathrm{p} 65$ subunit of $\mathrm{NF}-\kappa \mathrm{B}$ into the nucleus in response to tumor necrosis factor (TNF)- $\alpha$, a key indication of pathway activity. Translocation of p65 was dose dependently attenuated in APX2009- and APX2014-treated HRECs (Fig. 6A). Moreover, production of the mRNA of VEGFA, VCAM1, and CCL20, all of which are downstream of NF- $\kappa$ B, was decreased 3- to 10 -fold by these compounds (Fig. 6, B-D).

APX2009 and APX2014 Block Angiogenesis Ex Vivo. As a further test of antiangiogenic activity, we employed a choroidal sprouting assay using murine choroidal explants to test the effect of the APX compounds in a complex microvascular bed in tissues (Fig. 7). In this assay, choroidal cells grow out of the choroidal tissue piece into a surrounding Matrigel matrix. Both compounds significantly reduced sprouting, with APX2014 being more potent. At $10 \mu \mathrm{M}$, APX2009 reduced sprouting by $\sim 70 \%$ compared with control (Fig. 7, A and B), whereas at $1 \mu \mathrm{M}$ (the highest concentration tested), APX2014 reduced sprouting by $\sim 60 \%$ compared with control (Fig. 7, C and D).

Systemic Ref-1 Inhibition with Parent Compound APX3330 Can Prevent L-CNV. Previous efforts to attenuate ocular neovascularization by Ref-1 inhibition using APX3330 relied on intravitreal delivery of compound (Jiang et al., 2011; Li et al., 2014a,b). Although this is the delivery route of the standard-of-care anti-VEGF biologics and ensures that the drug gets to the right place, in humans it is laborintensive, causes patient discomfort, and incurs a risk of potentially vision-threatening endophthalmitis (Day et al., 2011). Thus, we explored whether systemic (intraperitoneal) administration of Ref- 1 inhibitors could offer an alternative route of therapy for L-CNV. As a proof-of-concept, we tested i.p. injections of the first-generation Ref-1 inhibitor APX3330 (7) delivered $50 \mathrm{mg} / \mathrm{kg}$ twice daily, 5 days on/2 days off, for 2 weeks. This dosing regimen was chosen as it was previously successful and nontoxic for preclinical tumor studies (Fishel et al., 2011; Lou et al., 2014; Biswas et al., 2015). Animals treated with APX3330 displayed significantly reduced L-CNV volume (Fig. 8). 
A

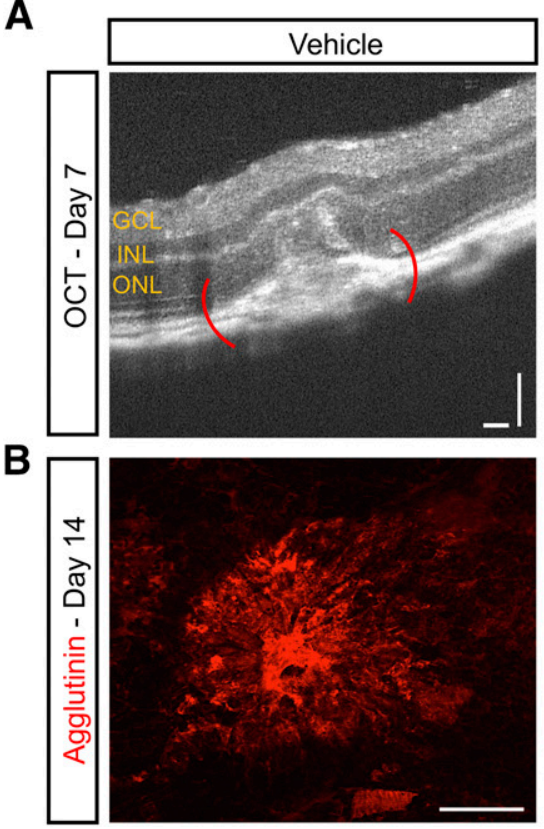

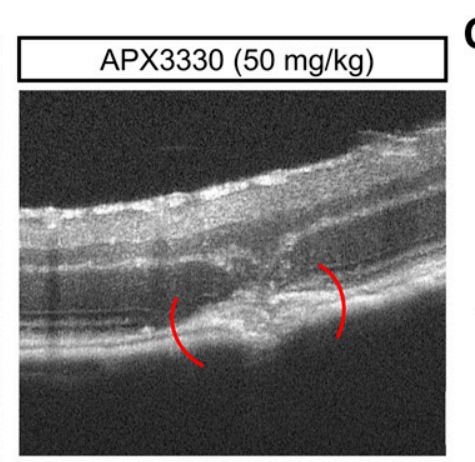

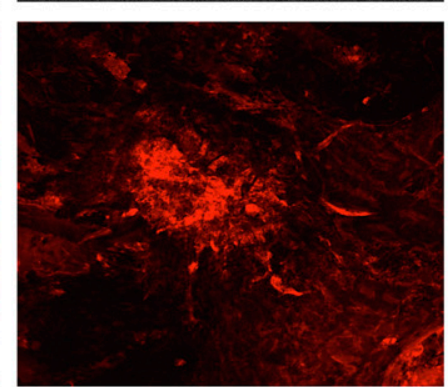

\section{C}

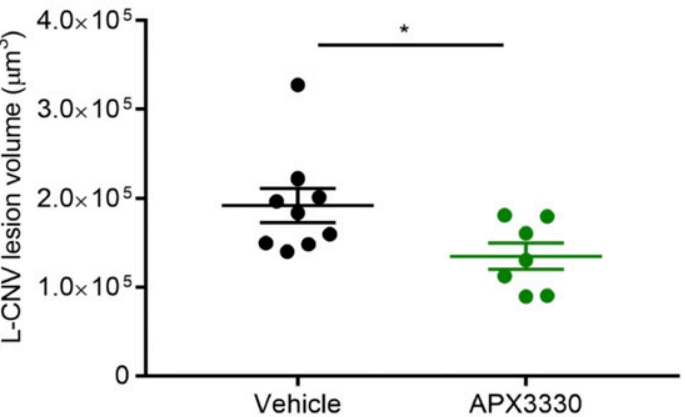

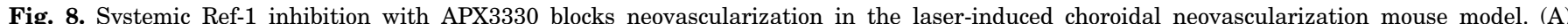

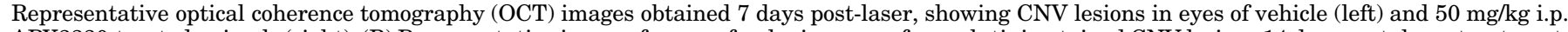

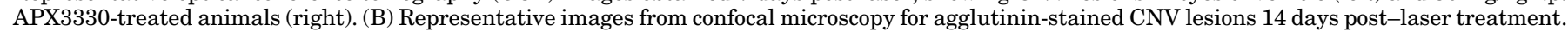

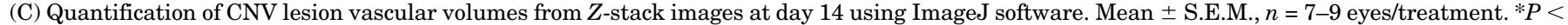
0.05 (unpaired Student's $t$ test). Scale bars, $100 \mu \mathrm{m}$. GCL, ganglion cell layer; INL, inner nuclear layer; ONL, outer nuclear layer.

Systemic Administration of More Potent Derivative APX2009 Reduces L-CNV Significantly. Given that APX3330 reduced L-CNV with systemic administration, we explored whether similar effects could be observed with our second-generation Ref-1 inhibitors. We chose APX2009 for this experiment as it had previously been safely dosed in animals (Kelley et al., 2016). We used two dosage regimens previously employed, 12.5- or $25-\mathrm{mg} / \mathrm{kg}$ twice daily for 2 weeks. The lower dose did not reduce L-CNV, but the $25 \mathrm{mg} / \mathrm{kg}$ dose had a marked effect (Fig. 9). This was qualitatively evident by OCT imaging on day 7, and even more substantial on day 14 (Fig. 9A). In addition, reduced fluorescein leakage was seen in lesions by fluorescein angiography at day 14 (Fig. 9B). Finally, L-CNV lesion volume assessed by ex vivo staining with agglutinin (Fig. 9C) and isolectin B4 (Supplemental Fig. 8), was reduced approximately 4-fold compared with vehicle by $25 \mathrm{mg} / \mathrm{kg}$ APX2009 (Fig. 9D).

\section{Discussion}

We have identified two new Ref-1 inhibitors, APX2009 and APX2014, and demonstrated antiangiogenic activity of these compounds. Moreover, we showed for the first time that systemic administration of Ref-1 inhibitors can attenuate L-CNV. As L-CNV is a widely-used model that recapitulates the choroidal neovascularization underlying wet AMD, Ref-1 inhibition could find therapeutic utility for this indication. Our in vitro data, and previous work (Jiang et al., 2011), suggest that Ref-1 inhibition also effectively blocks angiogenesis involving retinal endothelial cells. Thus, these inhibitors may also be useful for retinal neovascular diseases like ROP and PDR.

The observed effects are probably attributable to inhibition of Ref-1 redox signaling, rather than DNA repair inhibition, as the compounds are specific for redox signaling inhibition. The molecularly distinct functional portions of Ref-1, redox and DNA repair, are completely independent. For example, mutations of Ref-1/APE1 can abrogate the redox function without affecting DNA repair function, and vice versa (McNeill and Wilson, 2007; Vasko et al., 2011). Moreover, Ref-1 inhibitors such as APX3330 do not inhibit APE1 endonuclease activity. In fact, APX3330 and APX2009 can enhance APE1 repair activity in neurons (Kelley et al., 2016), potentially contributing to a neuroprotective effect of these agents, which could offer an added benefit in the context of photoreceptor cell death in neovascular eye diseases.

Given their anti-Ref-1 redox signaling activity, APX2009 and APX2014 probably exert their antiangiogenic effects by blocking the activation of transcription factors induced by Ref-1. This includes NF- $\mathrm{B}$ as shown, and also possibly HIF-1 $\alpha$; both of these can regulate VEGF (Forsythe et al., 1996). In retinal pigment epithelial cells, APX3330 reduced both NF- $\kappa$ B and HIF- $1 \alpha$ activity, with a concomitant reduction in VEGF expression (Li et al., 2014a,b), as we have shown here for APX2009 and APX2014. Additionally, APX3330 treatment of stroke in type 1 diabetes mellitus rats significantly decreased total vessel density and VEGF expression (Yan et al., 2018). However, the full spectrum of transcription factors modulated by Ref-1 inhibition in the context of ocular neovascularization remains to be determined.

We did not observe obvious intraocular or systemic toxicity of the two compounds tested in vivo (APX3330 and APX2009), nor did we see apoptosis or substantial cell death in migration, tube formation, and choroidal sprouting assays. These findings are consistent with the excellent safety profile for APX3330 in humans (Shah et al., 2017). Nonetheless, ocular toxicity of the new compounds and intraocular pharmacokinetics remain to be thoroughly examined. 
A

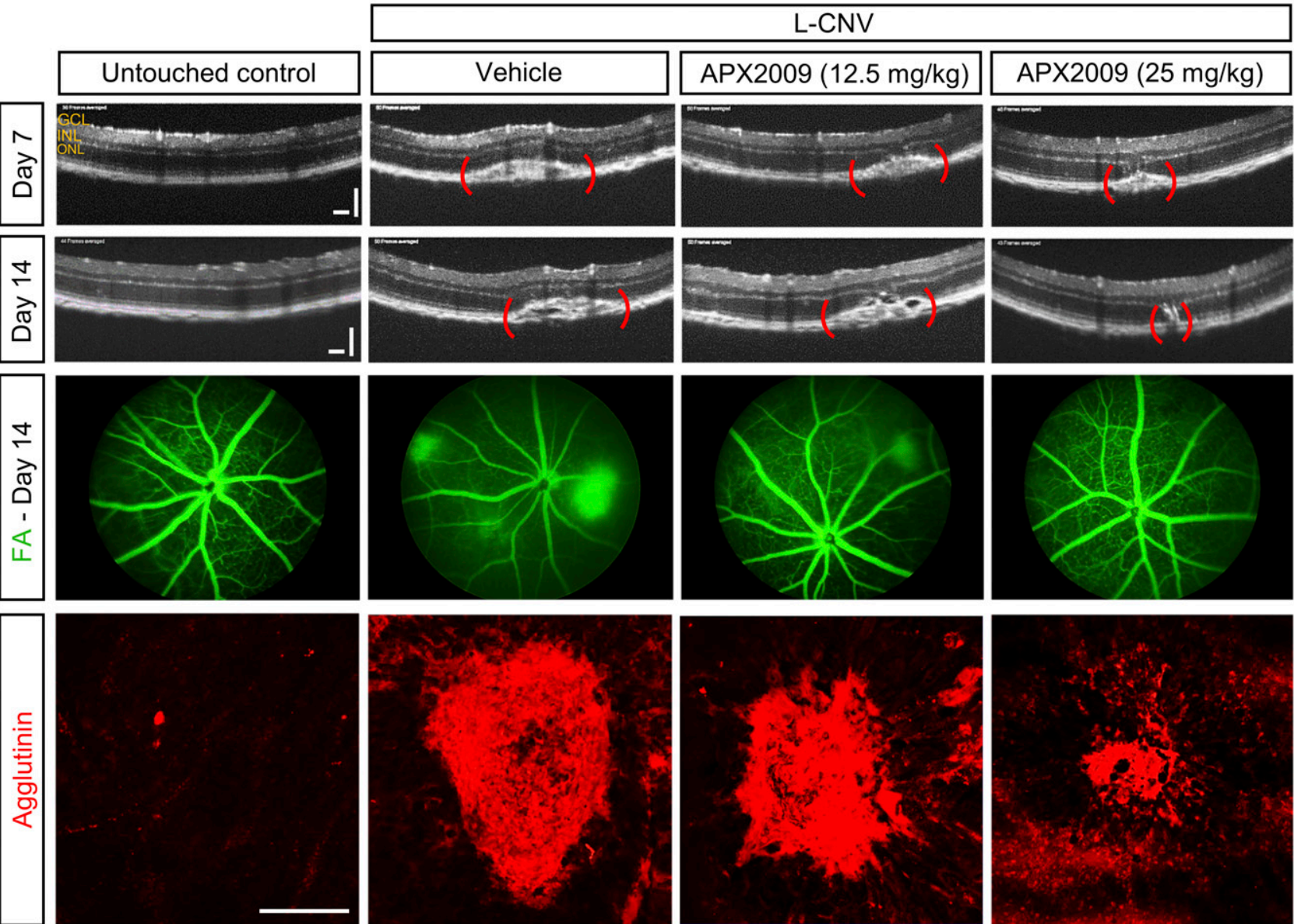

D

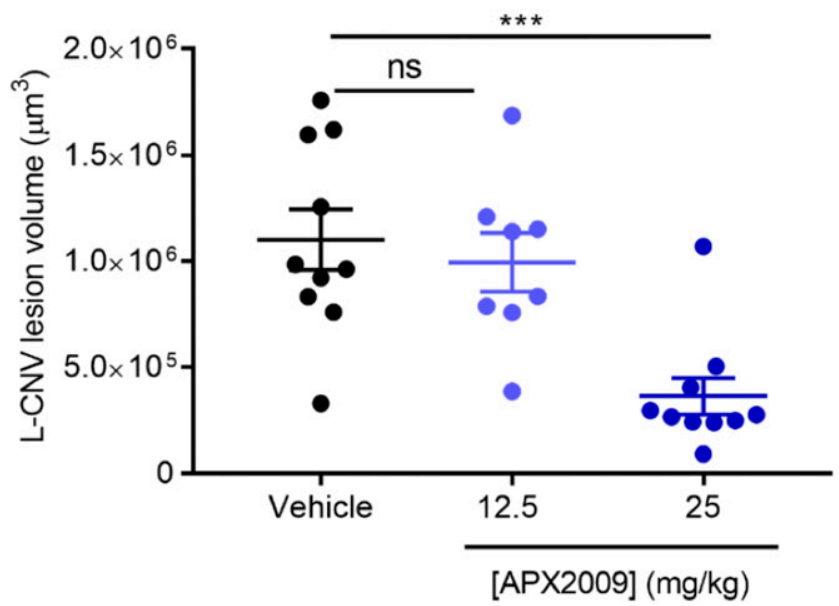

Fig. 9. Intraperitoneal APX2009 inhibits choroidal neovascularization in the L-CNV mouse model. (A) Representative OCT images obtained 7 and 14 days post-laser, showing CNV lesions of untouched control, vehicle, and 12.5 and $25 \mathrm{mg} / \mathrm{kg}$ APX2009 compound i.p. injected twice daily until 14 days post-laser treatment. (B) Fluorescein angiography (FA) of CNV showing the vascular leakage suppression by APX2009. (C) Representative images from confocal microscopy for agglutinin-stained CNV lesions 14 days post-laser treatment. (D) Quantification of CNV lesion vascular volumes from $Z$-stack images at day 14 using ImageJ software. Mean \pm S.E.M., $n=8-10$ eyes/treatment. ns, nonsignificant; *** $P<0.001$ compared with DMSO control (oneway ANOVA with Tukey's post-hoc test). Scale bar, $100 \mu \mathrm{m}$. GCL, ganglion cell layer; INL, inner nuclear layer; ONL, outer nuclear layer. See also Supplemental Fig. 8.

A well tolerated, systemic drug therapy has significant potential for treatment of neovascular eye diseases. The existing approved drugs are all biologics requiring intravitreal injection in an ophthalmologist's clinic. An orally bioavailable drug (as with APX3330) could be administered at home, potentially as a once daily pill. The tradeoff for such a therapy would be much more frequent dosing than that required for intravitreal injections (monthly or less), and more substantial systemic exposure than that seen with intravitreal therapies. Given the strong safety profile of Ref-1 inhibitors, this approach is feasible. Moreover, patient and healthcare system costs might be significantly lower with such a therapy, as office visits and injection procedures could be reduced. 
In summary, we have explored Ref- 1 inhibition as an ocular antiangiogenic therapy. We used two novel inhibitors of Ref-1 activity to demonstrate antiangiogenic effects in vitro, ex vivo, and in an animal model of choroidal neovascularization. The approach of targeting Ref-1 activity, and these small-molecule inhibitors in particular, holds promise for development of therapies for treating diseases such as wet AMD, PDR, and ROP.

\section{Acknowledgments}

We thank Rakshin Kharwadkar for assistance with proliferation assays.

\section{Authorship Contributions}

Participated in research design: Sardar Pasha, Sishtla, Sulaiman, Park, Shetty, Shah, Fishel, Kelley, Corson.

Conducted experiments: Sardar Pasha, Sishtla, Sulaiman, Park, Shetty, Shah.

Contributed new reagents or analytic tools: Kelley, Wikel.

Performed data analysis: Sardar Pasha, Sishtla, Sulaiman, Park, Shetty, Shah, Corson.

Wrote or contributed to the writing of the manuscript: Sardar Pasha, Sishtla, Sulaiman, Park, Shetty, Shah, Fishel, Wikel, Kelley, Corson.

\section{References}

Basavarajappa HD, Lee B, Fei X, Lim D, Callaghan B, Mund JA, Case J, Rajashekhar G, Seo SY, and Corson TW (2014) Synthesis and mechanistic studies of a novel homoisoflavanone inhibitor of endothelial cell growth. PLoS One 9:e95694.

Basavarajappa HD, Sulaiman RS, Qi X, Shetty T, Sheik Pran Babu S, Sishtla KL Lee B, Quigley J, Alkhairy S, Briggs CM, et al. (2017) Ferrochelatase is a therapeutic target for ocular neovascularization. EMBO Mol Med 9:786-801.

Biswas A, Khanna S, Roy S, Pan X, Sen CK, and Gordillo GM (2015) Endothelial cell tumor growth is Ape/ref-1 dependent. Am J Physiol Cell Physiol 309:C296-C307.

Campochiaro PA (2013) Ocular neovascularization. J Mol Med (Berl) 91:311-321.

Cardoso AA, Jiang Y, Luo M, Reed AM, Shahda S, He Y, Maitra A, Kelley MR, and Fishel ML (2012) APE1/Ref-1 regulates STAT3 transcriptional activity and APE1/Ref-1-STAT3 dual-targeting effectively inhibits pancreatic cancer cell survival. PLoS One 7:e47462.

Chiarini LB, Freitas FG, Petrs-Silva H, and Linden R (2000) Evidence that the bifunctional redox factor / AP endonuclease Ref-1 is an anti-apoptotic protein associated with differentiation in the developing retina. Cell Death Differ 7:272-281.

Day S, Acquah K, Mruthyunjaya P, Grossman DS, Lee PP, and Sloan FA (2011) Ocular complications after anti-vascular endothelial growth factor therapy in Medicare patients with age-related macular degeneration. Am J Ophthalmol 152: $266-272$.

Evans AR, Limp-Foster M, and Kelley MR (2000) Going APE over ref-1. Mutat Res 461:83-108.

Falavarjani KG and Nguyen QD (2013) Adverse events and complications associated with intravitreal injection of anti-VEGF agents: a review of literature. Eye (Lond) 27:787-794.

Fishel ML, Colvin ES, Luo M, Kelley MR, and Robertson KA (2010) Inhibition of the redox function of APE1/Ref-1 in myeloid leukemia cell lines results in a hypersensitive response to retinoic acid-induced differentiation and apoptosis. Exp Hematol 38:1178-1188.

Fishel ML, Jiang Y, Rajeshkumar NV, Scandura G, Sinn AL, He Y, Shen C, Jones DR, Pollok KE, Ivan M, et al. (2011) Impact of APE1/Ref-1 redox inhibition on pancreatic tumor growth. Mol Cancer Ther 10:1698-1708.

Fishel ML, Wu X, Devlin CM, Logsdon DP, Jiang Y, Luo M, He Y, Yu Z, Tong Y, Lipking KP, et al. (2015) Apurinic/apyrimidinic endonuclease/redox factor-1 (APE1/Ref-1) redox function negatively regulates NRF2. J Biol Chem 290: 3057-3068.

Forsythe JA, Jiang BH, Iyer NV, Agani F, Leung SW, Koos RD, and Semenza GL (1996) Activation of vascular endothelial growth factor gene transcription by hypoxia-inducible factor 1. Mol Cell Biol 16:4604-4613.

Grossniklaus HE, Kang SJ, and Berglin L (2010) Animal models of choroidal and retinal neovascularization. Prog Retin Eye Res 29:500-519.

Jedinak A, Dudhgaonkar S, Kelley MR, and Sliva D (2011) Apurinic/Apyrimidinic endonuclease 1 regulates inflammatory response in macrophages. Anticancer Res 31:379-385.

Jiang A, Gao H, Kelley MR, and Qiao X (2011) Inhibition of APE1/Ref-1 redox activity with APX3330 blocks retinal angiogenesis in vitro and in vivo. Vision Res 51: 93-100.

Kelley MR, Georgiadis MM, and Fishel ML (2010) DNA repair \& redox signaling, in The Tumor Microenvironment (Bagley $\mathrm{R}$ ed) pp 133-168, Springer Science+ Business Media, New York, NY.

Kelley MR, Georgiadis MM, and Fishel ML (2012) APE1/Ref-1 role in redox signaling: translational applications of targeting the redox function of the DNA repair/redox protein APE1/Ref-1. Curr Mol Pharmacol 5:36-53.

Kelley MR, Luo M, Reed A, Su D, Delaplane S, Borch RF, Nyland RL, II, Gross ML and Georgiadis MM (2011) Functional analysis of novel analogues of E3330 that block the redox signaling activity of the multifunctional AP endonuclease/redox signaling enzyme APE1/Ref-1. Antioxid Redox Signal 14:1387-1401.

Kelley MR and Wikel JH (2015) inventors, Indiana University Research and Technology Corporation, ApeX Therapeutics, Inc., assignees. Quinone compounds for treating Ape1 mediated diseases. U.S. patent 9,193,700. 2015 Nov 24

Kelley MR, Wikel JH, Guo C, Pollok KE, Bailey BJ, Wireman R, Fishel ML, and Vasko MR (2016) Identification and characterization of new chemical entities targeting apurinic/apyrimidinic endonuclease 1 for the prevention of chemotherapy-induced peripheral neuropathy. J Pharmacol Exp Ther 359:300-309.

Lando D, Pongratz I, Poellinger L, and Whitelaw ML (2000) A redox mechanism controls differential DNA binding activities of hypoxia-inducible factor (HIF) 1alpha and the HIF-like factor. J Biol Chem 275:4618-4627.

Li L, Cheung SH, Evans EL, and Shaw PE (2010) Modulation of gene expression and tumor cell growth by redox modification of STAT3. Cancer Res 70:8222-8232.

Li Y, Liu X, Zhou T, Kelley MR, Edwards P, Gao H, and Qiao X (2014a) Inhibition of APE1/Ref-1 redox activity rescues human retinal pigment epithelial cells from oxidative stress and reduces choroidal neovascularization. Redox Biol 2:485-494.

Li Y, Liu X, Zhou T, Kelley MR, Edwards PA, Gao H, and Qiao X (2014b) Suppression of choroidal neovascularization through inhibition of APE1/Ref-1 redox activity. Invest Ophthalmol Vis Sci 55:4461-4469.

Logsdon DP, Grimard M, Luo M, Shahda S, Jiang Y, Tong Y, Yu Z, Zyromski N, Schipani E, Carta F, et al. (2016) Regulation of HIF1 $\alpha$ under hypoxia by APE1/Ref1 impacts CA9 expression: dual targeting in patient-derived 3D pancreatic cancer models. Mol Cancer Ther 15:2722-2732.

Lou D, Zhu L, Ding H, Dai HY, and Zou GM (2014) Aberrant expression of redox protein Ape1 in colon cancer stem cells. Oncol Lett 7:1078-1082.

Luo M, Delaplane S, Jiang A, Reed A, He Y, Fishel M, Nyland RL, II, Borch RF, Qiao $\mathrm{X}$, Georgiadis MM, et al. (2008) Role of the multifunctional DNA repair and redox signaling protein Ape1/Ref-1 in cancer and endothelial cells: small-molecule inhibition of the redox function of Ape1. Antioxid Redox Signal 10:1853-1867.

Luo M, Zhang J, He H, Su D, Chen Q, Gross ML, Kelley MR, and Georgiadis MM (2012) Characterization of the redox activity and disulfide bond formation in apurinic/apyrimidinic endonuclease. Biochemistry 51:695-705.

Lux A, Llacer H, Heussen FM, and Joussen AM (2007) Non-responders to bevacizumab (Avastin) therapy of choroidal neovascular lesions. Br J Ophthalmol 91:1318-1322.

McIlwain DW, Fishel ML, Boos A, Kelley MR, and Jerde TJ (2017) APE1/Ref-1 redoxspecific inhibition decreases survivin protein levels and induces cell cycle arrest in prostate cancer cells. Oncotarget 9:10962-10977.

McNeill DR and Wilson DM, III (2007) A dominant-negative form of the major human abasic endonuclease enhances cellular sensitivity to laboratory and clinical DNA-damaging agents. Mol Cancer Res 5:61-70.

Nishi T, Shimizu N, Hiramoto M, Sato I, Yamaguchi Y, Hasegawa M, Aizawa S, Tanaka H, Kataoka K, Watanabe $\mathrm{H}$, et al. (2002) Spatial redox regulation of a critical cysteine residue of NF-kappa B in vivo. J Biol Chem 277:44548-44556.

Poor SH, Qiu Y, Fassbender ES, Shen S, Woolfenden A, Delpero A, Kim Y, Buchanan N, Gebuhr TC, Hanks SM, et al. (2014) Reliability of the mouse model of choroida neovascularization induced by laser photocoagulation. Invest Ophthalmol Vis Sci 55:6525-6534.

Prasad PS, Schwartz SD, and Hubschman JP (2010) Age-related macular degeneration: current and novel therapies. Maturitas 66:46-50.

Seo YR, Kelley MR, and Smith ML (2002) Selenomethionine regulation of p53 by a ref1-dependent redox mechanism. Proc Natl Acad Sci USA 99:14548-14553.

Shah F, Logsdon D, Messmann RA, Fehrenbacher JC, Fishel ML, and Kelley MR (2017) Exploiting the Ref-1-APE1 node in cancer signaling and other diseases: from bench to clinic. NPJ Precis Oncol 1:19.

Su DG, Delaplane S, Luo M, Rempel DL, Vu B, Kelley MR, Gross ML, and Georgiadis MM (2011) Interactions of apurinic/apyrimidinic endonuclease with a redox inhibitor: evidence for an alternate conformation of the enzyme. Biochemistry 50:82-92.

Sulaiman RS, Merrigan S, Quigley J, Qi X, Lee B, Boulton ME, Kennedy B, Seo SY, and Corson TW (2016) A novel small molecule ameliorates ocular neovascularisation and synergises with anti-VEGF therapy. Sci Rep 6:25509.

Sulaiman RS, Quigley J, Qi X, O'Hare MN, Grant MB, Boulton ME, and Corson TW (2015) A simple optical coherence tomography quantification method for choroidal neovascularization. J Ocul Pharmacol Ther 31:447-454.

Vasko MR, Guo C, Thompson EL, and Kelley MR (2011) The repair function of the multifunctional DNA repair/redox protein APE1 is neuroprotective after ionizing radiation. DNA Repair (Amst) 10:942-952.

Wenzel AA, O'Hare MN, Shadmand M, and Corson TW (2015) Optical coherence tomography enables imaging of tumor initiation in the TAg-RB mouse model of retinoblastoma. Mol Vis 21:515-522.

Xanthoudakis S and Curran T (1992) Identification and characterization of Ref-1, a nuclear protein that facilitates AP-1 DNA-binding activity. EMBO J 11:653-665. Xanthoudakis S, Miao G, Wang F, Pan YC, and Curran T (1992) Redox activation of FosJun DNA binding activity is mediated by a DNA repair enzyme. EMBO $J$ 11:3323-3335

Yan T, Venkat P, Chopp M, Zacharek A, Yu P, Ning R, Qiao X, Kelley MR, and Chen $J$ (2018) APX3330 promotes neurorestorative effects after stroke in type one diabetic rats. Aging Dis 9:453-466.

Zhang J, Luo M, Marasco D, Logsdon D, LaFavers KA, Chen Q, Reed A, Kelley MR, Gross ML, and Georgiadis MM (2013) Inhibition of apurinic/apyrimidinic endonuclease I's redox activity revisited. Biochemistry 52:2955-2966.

Zou GM, Karikari C, Kabe Y, Handa H, Anders RA, and Maitra A (2009) The Ape-1/ Ref-1 redox antagonist E3330 inhibits the growth of tumor endothelium and endothelial progenitor cells: therapeutic implications in tumor angiogenesis. $J$ Cell Physiol 219:209-218.

Address correspondence to: Dr. Timothy W. Corson, Eugene and Marilyn Glick Eye Institute, Department of Ophthalmology, Indiana University School of Medicine, 1160 West Michigan Street, Indianapolis, IN 46202. E-mail: tcorson@iu.edu 